\title{
Riesgos y consecuencias de las prácticas sexuales en los adolescentes de Juigalpa, bajo los efectos de alcohol y otras drogas, Chontales, Nicaragua
}

Francis Raquel Gallardo Bravo ${ }^{1}$

1 UNAN- Managua, FAREM-Chontales, Correo Electrónico: rgallardo1975@gmail.com

\section{RESUMEN}

En cuanto a las variables de educación sobre SIDA y conversación con sus padres sobre el SIDA, el colegio es la institución que de manera regular esta en contacto con la mayoría de los adolescentes, que 321 de los adolescentes conocen sobre el SIDA que han recibido en el colegio, y 228 estudiantes conversaron con sus padres en relación al SIDA. En el medio familiar, la información sobre SIDA, son temas menos hablados por los padres, probable serias por tiempo, por comunicación, por falta de información que ni ellos la tienen. La variable sexual activa un porcentaje importante dice haber iniciado la vida sexual, pero un porcentaje mayor no lo ha hecho, probablemente tengan miedo de cómo evitar un embarazo no deseado, o no tienen novio (a), puede ser por los aspectos sociales como la familia. En cuanto a la variable edad de inicio de la vida sexual según sexo, los varones suelen iniciar su vida sexual activa, antes que las mujeres, pero ambos en menor o mayor medida están expuestos a los riesgos que implican llevar una sexualidad sin cuidos. Los adolescentes tienen su primera relación sexual a edades cada vez menores, y si bien físicamente están absolutamente preparados mentalmente, no siempre están listos para lidiar con las consecuencias y las responsabilidades que eso genera. La variable cantidad de personas con quien ha tenido relaciones sexuales según sexo, el sexo femenino es el que representa menor el porcentaje en cuanto número de personas que han tenido relaciones sexuales, esto probablemente se cuidan de su salud, adquieren conciencia acerca de las responsabilidades de tener hijos, en cambio en el sexo masculino el porcentaje es un poco mayor debido al libertinaje que tiene el varón y además el varón dice que no ha tenido relaciones con más personas, apenas un $6 \%$ dice si haber tenido relaciones con más personas.

En relación a las variables has consumido alcohol, droga en la última relación sexual, usaste condón en la última relación sexual, que método usaron para evitar embarazo en la última relación sexual, los adolescentes de Juigalpa consumen muy poca droga para las relaciones sexuales, esto quiere decir que la mayoría de ellos están conscientes de las cosas que hacen, los adolescentes estudiados la gran mayoría utilizan condón es sus relaciones sexuales, esto quiere decir que se protegen de embarazo no deseados y de algunas enfermedades de transmisión sexual, el condón es el que prevalece en las relaciones sexuales, los adolescentes están seguros que el condón es para evitar embarazo. En la variable te han dicho alguna vez un médico o enfermera que tenías enfermedad de transmisión sexual, la prevalencia de ambos sexos es baja, esto quiere decir que los adolescentes estudiados se chequean a menudo, de 340 adolescentes u solo 10 adolescentes refieren haber tenido enfermedad de transmisión sexual, en cuanto a los adolescentes que tienen vida sexual activa es muy baja la prevalencia de infecciones de transmisión sexual, de 102 adolescentes con vida sexual, refirió 7 adolescentes haber tenido enfermedad de transmisión sexual.

Palabras Claves: sexualidad, género, adolescentes, drogas, embarazo, enfermedad. 


\title{
Risks and consequences of sexual practices among adolescents in Juigalpa, under the influence of alcohol and other drugs, Chontales, Nicaragua
}

1 UNAN- Managua, FAREM-Chontales, E-mail: rgallardo1975@gmail.com

Francis Raquel Gallardo Bravo ${ }^{1}$

\begin{abstract}
As for the variables of AIDS education and conversation with their parents about AIDS, the school is the institution that regularly in contact with most adolescents, 321 adolescents know about AIDS that have received school, and 228 students talked with their parents in relation to AIDS. In the family environment, information on AIDS issues is less spoken by parents, probably serious by time, communication, lack of information or they have it. Sexual active variable a significant percentage said to have initiated sexual activity, but a higher percentage has not done so, probably afraid of how to avoid an unwanted pregnancy, or have boyfriends (a), may be for the social aspects as family. As for the variable age of onset of sexual activity by sex, men tend to begin sexual life earlier than women, but both to a lesser or greater extent exposed to the risks involved lead sexuality without oversights. Teens have their first sexual intercourse at younger and younger ages, and although they are physically quite prepared mentally, they are not always ready to deal with the consequences and responsibilities that generates. The variable number of people who have had sex by sex, the female sex is represented as the percentage lower number of people who have had sex, this will probably take care of your health, become aware about the responsibilities of have children, whereas in males the percentage is a bit higher because of the debauchery that is the man and also the man says he has not had sex with more people, only $6 \%$ say if having sex with more people.
\end{abstract}

Regarding the variables you have consumed alcohol, drugs at last sex, you used a condom at last sex , which method used to prevent pregnancy at last sex, teens Juigalpa consume very little drug for sex , this means that most of them are aware of the things they do, the vast majority adolescents studied condom use is sexual intercourse, this means that protect against unwanted pregnancy and some STIs, the condom is the prevailing in sex, teens are sure that the condom is to prevent pregnancy. In the variable you ever a doctor or nurse that you had a sexually transmitted disease have said, the prevalence of both sexes is low, this means that the adolescents studied are checked often, of 340 teenagers or only 10 teens report having had disease sexually transmitted about teens who are sexually active is very low prevalence of sexually transmitted infections, of 102 sexually active adolescents, said seven teenagers have had a sexually transmitted disease

Keywords: sexuality, gender, adolescents, drugs, pregnancy, disease. 


\section{INTRODUCCIÓN}

La sexualidad, y el consumo de alcohol y drogas en los adolescentes, figuran en la actualidad como uno de los asuntos con mayor prioridad para la salud pública. La preocupación radica en reducir los riesgos de infecciones de transmisión sexual, el virus de la inmunodeficiencia humana y los embarazos tempranos no deseados, Castaño (2013).

Tener prácticas sexuales no protegidas, y el consumo de sustancias psicoactivas en los jóvenes, son dos de las mayores preocupaciones de la salud publica en la actualidad. En América Latina, la OPS estima que la mitad de los nuevos casos por VIH se reportan en personas con edad inferior a 25 años, los cuales se han contaminado por vía sexual. En los Estados Unidos los mayores índices de contagio con el $\mathrm{VIH}$ están en los jóvenes con edades entre 17 y 19 años, en su mayoría varones, Castaño (2013).

Método: Se realizó un estudio descriptivo de corte transversal, en el cual participaron estudiantes activos de los tres colegios de Juigalpa, Chontales, Nicaragua, con el fin de medir la prevalencias de las conductas que ponen en riesgo la salud de estos jóvenes.

Tomando como referencia la información de las matriculas del año 2012 de los participantes de estudio; se incluyeron estudiantes de primero a quinto año, con una población de 1,010 estudiantes.

Los estudiantes de los tres colegios, de las modalidades de vespertino. La muestra se seleccionó estratificado por cada colegio, los participantes del estudio se eligieron aleatoriamente en las aulas de primero a quinto año de los tres centros educativos, dichos aulas constan de 35 a 50 estudiantes (Femeninos y Masculinos), al momento de realizar la visita se tomó a los estudiantes que estaban presentes en cada colegio, obtenido las siguientes muestras parciales, C1, $94 / 350=25 \%, C 2,102 / 350=29 \%$, C3,
$154 / 350=50 \%$. Correspondiendo la muestra total (350/1010) para un 35\%.

La obtención de los datos se realizó mediante un cuestionario anónimo, autoadministrado, de fácil comprensión, integrado por preguntas cerradas. Se procedió a coordinar con la dirección del centro, para la aplicación del cuestionario en cada aula de clase, se utilizó el horario de clase y establecido para la aplicación del cuestionario, el cual fue distribuido en cada sección previa las explicaciones pertinentes para su llenado. Durante este proceso se aclararon algunas dudas a quienes lo requirieron entregando al final cada estudiante su cuestionario y depositándolo en una caja, el llenado duro media hora. Para la recolección de la información se utilizó el cuestionario sobre conductas de riesgo en adolescentes, elaborado por Centeno J. (1993).

El plan de análisis se procedió a realizar frecuencia simple por cada variable de las conductas de riesgo, además análisis de dos variables para la comparación de los colegios, sexo y otras variables de interés, en algunos casos se calculó el valor de $\mathrm{P}$, chi cuadrado y también se midió los ODDS RATIO en la búsqueda de algún tipo de asociación con las conductas de riesgo, estos cálculos fueron excepcionales; ya que el estudio es descriptivo. El análisis principal estuvo a cargo de establecer las medidas de prevalencia para las conductas de riesgo.

En cuanto a los aspectos éticos se solicitó el consentimiento informado a las autoridades del centro educativo y a los participantes del estudio, se notificó que la información se utilizaría exclusivamente con fines educativos y se garantizó la confidencialidad en la información a los encuestados y al derecho de no participar y de retirarse de la investigación en cualquier momento. 


\section{MATERIALES Y MÉTODOS}

\section{Instrumento}

Para la recolección de la información se utilizó el cuestionario sobre conductas de riesgo en adolescentes, elaborado por Centeno J. (1993)

Se procedió a realizar frecuencia simple por cada variable de las Conductas de Riesgo, además análisis de dos variables para la comparación de los colegios, sexo y otras variables de interés, en algunos casos se calculó el valor de $\mathrm{P}$, de Chi cuadrado y también se midió los ODDS RATIO en la búsqueda de algún tipo de asociación con las conductas de riesgo estos cálculos fueron excepcionales; ya que el estudio es descriptivo. El análisis principal estuvo a cargo de establecer las medidas de prevalencia para las Conductas de Riesgo.

\section{Procedimiento}

Se solicitó el consentimiento informado a las autoridades del centro educativo y a los participantes del estudio, se notificó que la información se utilizaría exclusivamente con fines educativos y se garantizó la confidencialidad en la información a los encuestados y al derecho de no participar y de retirarse de la investigación en cualquier momento.

La obtención de los datos se realizó mediante un cuestionario, autoadministrado, de fácil comprensión, integrado por preguntas cerradas. Se procedió a coordinar con la dirección del centro, para la aplicación del cuestionario en cada aula de clase, se utilizó el horario de clase ya establecido para la aplicación del cuestionario, el cual fue distribuido en cada sección previa las explicaciones pertinentes para su llenado. Durante este proceso se aclararon algunas dudas a quienes lo requirieron entregando al final cada estudiante su cuestionario y depositándolo en una caja. El llenado duro alrededor de media hora.
Se realizó un estudio descriptivo de corte transversal, en el cual participaron estudiantes activos de los colegios, C1, C2, C3 de la ciudad de JuigalpaChontales, con el fin de medir las prevalencias de las conductas que ponen en riesgo la salud de estos jóvenes.

Tomando como referencia la información de las matriculas del año 2012 de los colegios participantes de estudio; se incluyeron estudiantes de primero a quinto año, con una población de 1,010 estudiantes.

La muestra se seleccionó estratificada por cada colegio, los participantes del estudio se eligieron aleatoriamente en las aulas de primero a quinto años de los colegios, dichas aulas constan de 35 a 50 estudiantes (Femenino y Masculino), al momento de realizar la visita se tomó a los estudiantes que estaban presentes en cada colegio, obteniendo las siguientes muestras parciales, C1 $94 / 350=25 \%$, C2 $102 / 350=29 \%$, C3 154/350 = 50\%. Correspondiendo a la muestra total (350/1010) para un $35 \%$.

\section{RESULTADOS}

Cuadro S-1:

Educación sobre Sida en el colegio y Conversación sobre Sida con los padres.

\begin{tabular}{|c|c|c|c|c|}
\hline S.I.D.A. & SI (\%) & NO (\%) & $\begin{array}{l}\text { NO ME } \\
\text { ACUERDO } \\
(\%)\end{array}$ & TOTAL \\
\hline $\begin{array}{l}\text { 1. Educación sobre el SIDA } \\
\text { en el colegio }\end{array}$ & $321(92 \%)$ & $17 \quad(5 \%)$ & $11(3 \%)$ & 349 \\
\hline $\begin{array}{l}\text { 2. Conversación sobre el } \\
\text { SIDA con los padres }\end{array}$ & $228(66 \%)$ & 91 (26\%) & $28 \quad(8 \%)$ & 347 \\
\hline
\end{tabular}

Fuente: elaborado por el investigador.

En relación a la variable educación sobre SIDA en el colegio y conversación sobre SIDA con los padres, refleja que el $92 \%$ en el colegio le han dado educación sobre SIDA, y un $66 \%$ han conversado con sus padres sobre SIDA, un $26 \%$ refleja que no conversan con sus 
padres sobre el SIDA, seguido de un $8 \%$ que no se acuerdan haber conversado con sus padres sobre SIDA. En cuanto a la variable estudiantes ya iniciaron vida sexual, un $30 \%$ contestaron que si iniciaron la vida sexual activa, seguido de un $70 \%$ no han iniciado la vida sexual.

\section{Gráfico S-1:}

\section{Estudiantes que ya iniciaron Vida Sexual}

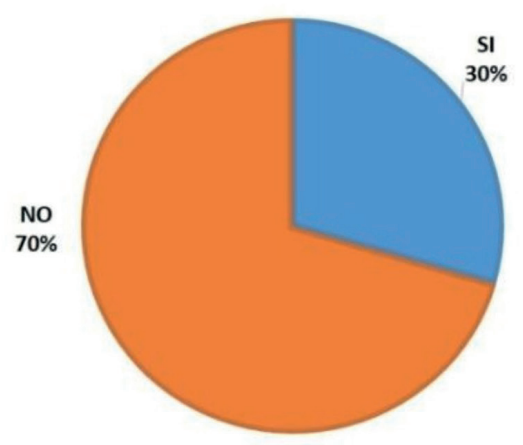

Fuente: elaborado por el investigador.

En relación a la variable edad de inicio de la vida sexual por sexo, la mayoría de los hombres lo hacen a los 14 años, en cambio la mayoría de mujeres lo hacen a los 15 .

\section{Grâfico S-2:}

Edad de Inicio de Vida Sexual según sexo. $(n=103)$

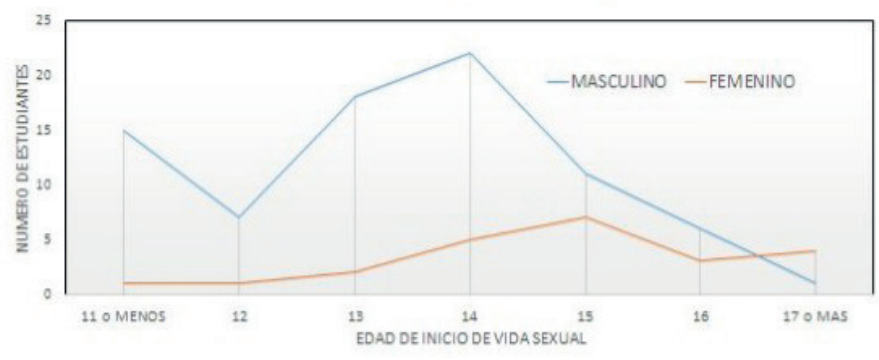

Fuente: elaborado por el investigador.

En casi todas las edades predominan la mayoría de hombres que iniciaron vida sexual sobre la cantidad de mujeres.

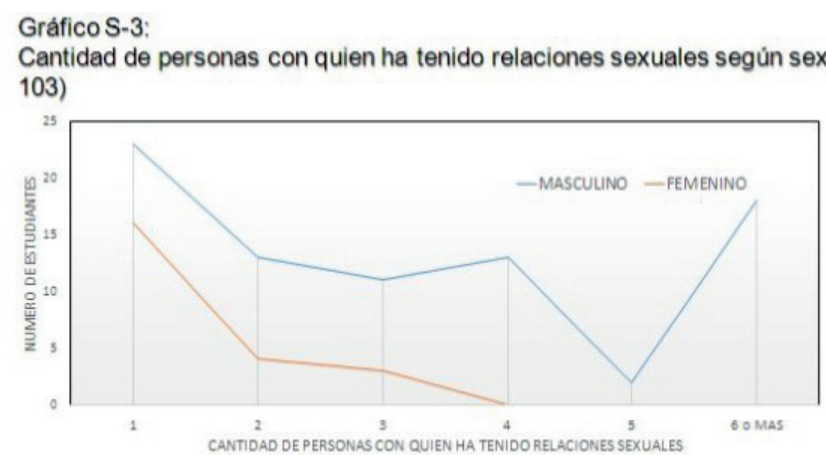

Fuente: elaborado por el investigador.

El $12 \%$ de las mujeres ya inicio vida sexual, en cambio en los hombres ya lo hizo la mitad, el 15\%. En relación a la variable cantidad de personas con quien ha tenido relaciones sexuales según sexo, en el sexo Masculino se observa un predominio sobre las mujeres. El sexo Femenino no registró más de 4 personas con quienes han tenido relaciones, en cambio los hombres llegan a 6 y más.

\section{Cuadro S-2:}

Otros aspectos de las relaciones sexuales

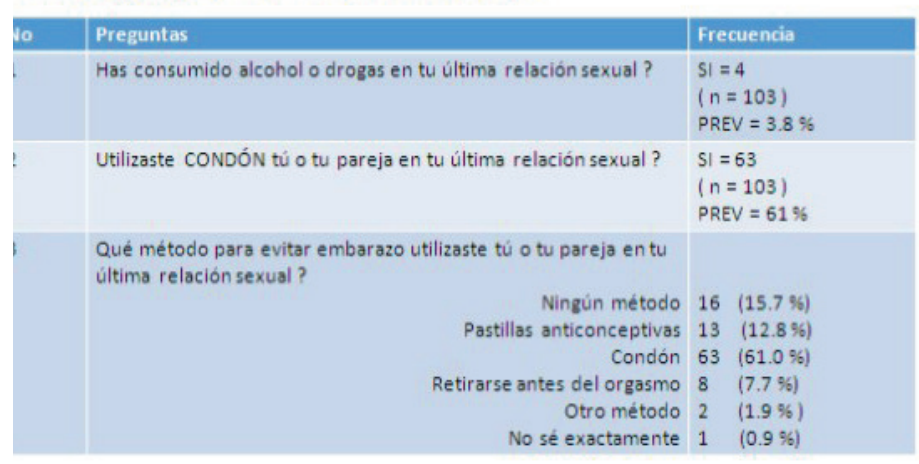

Fuente: elaborado por el investigador.

En relación a otros aspectos de las relaciones sexuales, el $3.8 \%$ de quienes han tenido relaciones $(n=103)$ dice que han consumido alcohol o drogas en la última relación. En cuanto al uso de condón tu o tu pareja en la última relación sexual, el 6.5\% afirma que si uso condón en la última relación sexual. En relación a cual método utilizaron tu o tu pareja para evitar un embarazo en la última relación sexual, el 16\% dice no uso ningún método, el $13 \%$ dice, que uso pastillas 
anticonceptivas, el $61 \%$ uso condón, el $8 \%$ se retiró antes del orgasmo, el $12 \%$ uso otro método, y el $1 \%$ no sabe exactamente que método uso.

\section{Cuadro S-3:}

Relaciones sexuales y embarazo

Pregunta: Cuantas veces has estado embarazada o Tú has dejado embarazada a alguien ?

\begin{tabular}{|c|c|c|c|}
\hline \multirow[t]{2}{*}{ Número de veces } & \multicolumn{2}{|c|}{ SEXO } & \multirow[t]{2}{*}{ TOTAL } \\
\hline & Femenino & Masculino & \\
\hline $\begin{array}{l}\text { UNA VEZ } \\
\text { DOS VECES O MAS } \\
\text { NO SE EXACTAMENTE } \\
\text { NINGUNA VEZ }\end{array}$ & $\begin{array}{c}2 \\
0 \\
0 \\
21\end{array}$ & $\begin{array}{c}4 \\
2 \\
3 \\
71\end{array}$ & $\begin{array}{l}6 \\
2 \\
3 \\
92\end{array}$ \\
\hline TOTAL & 23 & 80 & 103 \\
\hline
\end{tabular}

Fuente: elaborado por el investigador.

En cuanto a la variable número de veces que has estado embarazada (si es mujer) o tú has dejado embarazada a alguien (si es hombre), el 6\% dice que 1 vez, casi el $2 \%$ dice que 2 veces o más, el $3 \%$ dice que no sabe exactamente embarazo, pero el $89 \%$ dice ninguna vez.

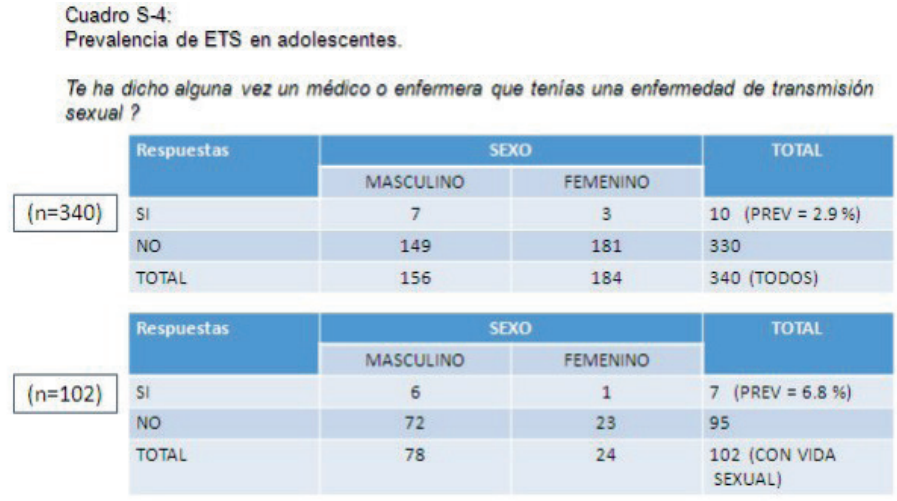

Fuente: elaborado por el investigador.

En la variable te ha dicho alguna ver un médico o enfermera que tenías una enfermedad de transmisión sexual, el $5 \%$ del sexo masculinos refiere que si ha tenido enfermedad de transmisión sexual, seguido del $2 \%$ del sexo femenino refiere que si ha tenido enfermedades de transmisión sexual, pero los adolescentes estudiados que tienen vida sexual activa, refiere que el $7 \%$ de hombres dice que si ha tendí enfermedad de transmisión sexual, y $4 \%$ de las mujeres.

Cuadro S-5:

Homosexualidad y Violación según Sexo

\begin{tabular}{|c|c|c|c|}
\hline \multirow[t]{2}{*}{ Homosexualidad } & \multicolumn{2}{|c|}{ SEXO } & \multirow[t]{2}{*}{ TOTAL } \\
\hline & MASCULINO & FEMENINO & \\
\hline SI & 6 & 5 & $11($ PREV $=3.2 \%)$ \\
\hline NO & 146 & 180 & 326 \\
\hline TOTAL & $152^{\prime \prime}$ & $185^{*}$ & $337^{*}$ \\
\hline \multirow{2}{*}{$\begin{array}{l}\text { Ha sufrido } \\
\text { VIOLACION }\end{array}$} & \multicolumn{2}{|c|}{ SEXO } & \multirow[t]{2}{*}{ TOTAL } \\
\hline & MASCULINO & FEMENINO & \\
\hline SI & $1^{* *}$ & $7^{* *}$ & $8^{* *}($ PREV $=2.4 \%)$ \\
\hline NO & 153 & 174 & 327 \\
\hline TOTAL & $154^{*}$ & $181^{*}$ & $335^{*}$ \\
\hline
\end{tabular}

Fuente: elaborado por el investigador.

En relación a la variable homosexualidad y violación según sexo, el $4 \%$ del sexo masculino dice que si es homosexual, en cambio el $96 \%$ asegura que no son homosexuales, el $3 \%$ de los que contestaron que si son homosexuales, la prevalencia de ambos sexos es de $3.2 \%$ de los que contestaron si son homosexuales, la variable ha sufrido violación, el $0.6 \%$ del sexo masculino dice que si ha sufrido violación, el 3.8\% del sexo femenino aseguran que sí, para un total de 335 adolescentes estudiados, con una prevalencia del $2.4 \%$ de ambos sexos de los que han sufrido violación.

\section{Cuadro S-6: \\ Edad de las violaciones}

\begin{tabular}{|c|l|l|}
\hline CASOS $(\mathbf{n}=8)$ & & GRUPO DE EDAD \\
\hline Cantidad & $\%$ & \\
\hline 5 & 62.5 & 10 Años o menos \\
\hline 1 & 12.5 & 13 a 14 Años \\
\hline 1 & 12.5 & 15 a 16 Años \\
\hline 1 & 12.5 & 17 a 18 Años \\
\hline 8 & 100 & \\
\hline
\end{tabular}

Fuente: elaborado por el investigador.

En cuanto a la edad de las violaciones, de los 8 casos de violación el $63 \%$ refiere a las edades de 10 años 
o menos, seguido de un $13 \%$ de 13 a 14 años, 1516 años y 17 a 18 años.

\begin{tabular}{|c|c|c|c|c|c|}
\hline \multirow[t]{2}{*}{ ABORTO } & \multicolumn{4}{|c|}{ SEXO } & \multirow[t]{2}{*}{ TOTAL } \\
\hline & MASCULINO & $\%$ & FEMENINO & $\%$ & \\
\hline SI & 2 & 1.3 & 2 & 1.0 & 4 (PREV $=1.1 \%)$ \\
\hline NO & 153 & 98.7 & 183 & 99.0 & 336 \\
\hline TOTAL & 155 & 100 & 185 & 100 & 340 \\
\hline
\end{tabular}

Fuente: elaborado por el investigador.

Loa abortos provocados apenas un $1.1 \%$ reportaron haberse o haber provocado un aborto.

\section{CONCLUSIONES}

Un $30 \%$ de los adolescentes ya inicio vida sexual, siendo la mayoría los del sexo masculino, así también son los varones quienes han tenido más parejas sexuales. Solamente 11 adolescentes refieren que han estado vinculados a un embarazo.

La prevalencia de Enfermedad de Transmisión Sexual (ETS), fue de $2.9 \%$ en toda la población. La prevalencia de homosexualidad fue de $3.2 \%$ y del total de estudiados un $2.4 \%$ afirmaron haber sufrido una violación.

\section{BIBLIOGRAFÍA}

Acero A, Escobar Castellanos G. (2007) Factores de riesgos para violencia y homicidio juvenil. Revista Colombiana de Psiquiatría, vol. XXXVI/ No. 1

Castaño Pérez, PhD. Guillermo, Arango Tobón MSc. Eduardo, Morales Mesa MSc. Santiago, Rodríguez Bustamante MSc. Alexander, Montoya Montoya MSc. Carolina, Fundación Universitaria Luis amigo, Medellín, Colombia, revista cubana de pediatría. 2013; 85 (1): 36-50.

Centeno J, Desarrollo y Validación de un cuestionario sobre Conductas de Riesgo en escolares y adolescentes, UNAN-LEON, 1993.

Cerbon Héctor. vitae desarrollo humano consultores, Articulo manejo y soluciones de Conflictos.

Ingles CJ, Delgado B, Bautista R, Torregrosa MS, Espadas JP, García - Fernández JM, et al Factores Psicosociales relacionados con el consumo de alcohol y tabaco en adolescentes. International J ClinHealthPsychol. 2007.

Judez J, Marrijuan M. Ética de los consumos de drogas. En: VV. AA. Consumo y control de drogas. Reflexiones desde la ética. Madrid: Fundación de Ciencias de la Salud/ Fundación de ayuda contra la Drogadicción; 2004.

Martínez, Martin, A., López, J.N., J. S., \& Martin, M. Comportamiento de riesgos: Violencia, prácticas sexuales d e riesgos y consumo de drogas ilegales en la juventud. Madrid, España, Entinema, 1998.

Martin, A., Martínez, J. N., López, J.S, \& Martin, M. J. Comportamiento de riesgos: Violencia, prácticas sexuales de riesgos y consumo de drogas ilegales en la juventud. Madrid, España: Entinema. |1998.

Orientaciones Técnicas. Atención de adolescentes con problemas de salud mental, dirigida a los equipos de atención primaria de salud. Ministerio de salud de Chile, Subsecretaria de Salud Pública, división de Prevención y Control de enfermedades, departamento de salud mental, programa de salud del adolescente y Joven. 2009.

Subsecretaria de Salud Pública, división de prevención y control de enfermedades. Departamento de salud mental, programa de salud del adolescente y jóvenes. Orientaciones técnicas. Atención de adolescentes con problemas de salud mental. Dirigida a los equipos de atención primaria de salud. Ministro de salud, Colombia, 2009. y adolescentes, UNAN-LEON, 1993. 